\title{
Common mental disorders: falling through the gap
}

People with mental illness are more likely than the rest of the population to have physical health problems and to die prematurely. The Lancet Psychiatry Commission on protecting physical health in people with mental illness draws attention to this global inequality. ${ }^{1}$

An issue clearly highlighted by the Commission is that we know less about the physical health of people with common mental disorders, like depression and anxiety, compared to people with severe mental illnesses (SMI). The risk of physical health problems and premature mortality compared to the general population is higher for people with SMI than for those with common mental disorders. For example, the odds of all-cause mortality is 2.5 times higher than the general population for people with schizophrenia and 1.7 times higher for people with depression. ${ }^{1}$ However, an estimated 23 million people worldwide have schizophrenia, whereas 300 million people have depression. ${ }^{2}$ The population level impact in terms of life-years lost is therefore far higher in common mental disorders than SMI. The level of unmet need in physical healthcare in people with common mental disorders is not well quantified, but potentially eclipses the issues in SMI populations.

Several research questions on morbidity and premature mortality remain unanswered for common mental disorders. One is whether the mortality gap between people with and without common mental disorders is increasing. Despite mortality rates decreasing in the population overall, the mortality gap between people with and without SMI is growing. ${ }^{3}$ Possible explanations for this are that interventions for negative health behaviours have been less effective at engaging people with mental health problems, that medications treating mental health problems detrimentally affect physical health, and that health effects of periods of economic recession have been larger in more socioeconomically disadvantaged groups. Through any/all of these mechanisms we could hypothesise that the mortality gap is also increasing for people with CMD compared to the general population.

Another question is to what extent are people with common mental disorders at increased risk of infectious diseases compared to the general population? The Commission identified a lack of evidence on rates of infectious diseases among people with common mental disorders. In particular, there are few large nationally representative studies on the prevalence of different types of infectious diseases and few studies of common mental disorders and infectious diseases set in primary care, where the vast majority of people with depression are treated. For patients with SMI, HIV, hepatitis B and hepatitis $C$ are between two and six times more common than in the general population. ${ }^{4}$ One reason for this could be increased substance misuse among people with SMI. ${ }^{4}$ Drug use along with risky sexual behaviours ${ }^{5}$ are more common in people with depression and anxiety compared to the general population, and therefore we might expect similarly elevated rates of these infectious diseases in common mental disorders.

Third, could primary care interventions reduce the risk of physical health problems in people with common mental disorders? Interventions targeting lifestyle risk factors like smoking, ${ }^{6}$ have been adapted for people with SMI. There have been 27 meta-analyses of physical health interventions for people with SMI and the seven trials of lifestyle interventions identified by the Commission were all in SMI populations. ${ }^{1}$ The development or adaptation of lifestyle interventions for people with common mental disorders appears to have lagged behind. This is despite evidence that people with common mental disorders experience higher rates of many negative health behaviours than the general population. Around $30 \%$ of people with depression ${ }^{7}$ and $20 \%$ of people with schizophrenia ${ }^{8}$ are likely to have an alcohol problem during their lifetime. Around $30 \%$ to $40 \%$ of people with depression are current smokers ${ }^{9}$ (and 60\% to 70\% of people with schizophrenia). Young people with depression are also at increased risk of many negative health behaviours, ${ }^{10}$ which may have long-term health implications.

The 2018 WHO guidelines recommend lifestyle interventions as first-line strategies for the management of physical health (including weight management, cardiovascular risk reduction, and diabetes treatment and prevention) in adults with SMI. ${ }^{1}$ [A: cite the guidelines here not the 
Commission]. There is no similar recommendation for people with common mental disorders. Overlooking populations with common mental disorders seems surprising (and neglectful) given the high prevalence of anxiety and depression and the evidence of association with lifestyle risk factors, morbidity and premature mortality. It is unclear if people with common mental disorders require modified approaches to risk factor management in line with SMI populations, but given the similar challenges this group faces it is reasonable to hypothesise that interventions need to be tailored to account for mental health issues.

Closing the gap between our understanding of the physical health of people with common mental disorders and SMI should be a research priority. Whether mental illnesses cause, share common antecedents with, or complicate the treatment of physical health problems, improved care and prevention in people with common mental disorders could substantially reduce the prevalence of morbidity and burden on healthcare services. A vital need to improve healthcare in SMI populations, who are among the most vulnerable in society, remains. However, at a population and individual level, addressing the physical health needs of people with common mental disorders would have considerable benefit.

Gemma Lewis and Joseph Hayes

Division of Psychiatry, University College London, 149 Tottenham Court Road, London W1T 7NF (GL and JH).

Corresponding author: Gemma.lewis@ucl.ac.uk

Dr. Lewis has nothing to disclose.

Dr. Hayes reports a Wellcome Trust Clinical Research Career Development Fellowship (211085/Z/18/Z) and support from the NIHR UCLH Biomedical Research Centre.

\section{References}

1 Commission [Lancet will add details]

1 Walker ER, McGee RE, Druss BG. Mortality in Mental Disorders and Global Disease Burden Implications. JAMA Psychiatry 2015; 72 : 334.

2 World Health Organization. The global burden of disease: 2004 update 2008. 2008.

3 Hayes JF, Marston L, Walters K, King MB, Osborn DPJ. Mortality gap for people with bipolar disorder and schizophrenia: UK-based cohort study 2000-2014. Br J Psychiatry 2017; 211: 175-81.

4 Bauer-Staeb C, Jörgensen L, Lewis G, Dalman C, Osborn DPJ, Hayes JF. Prevalence and risk factors for HIV, hepatitis $B$, and hepatitis $C$ in people with severe mental illness: a total population study of Sweden. The lancet Psychiatry 2017; 4: 685-93.

5 Cunningham K, Martinez DA, Scott-Sheldon LAJ, Carey KB, Carey MP, MASH Research Team. Alcohol Use and Sexual Risk Behaviors among Adolescents with Psychiatric Disorders: A Systematic Review and Meta-Analysis. J Child Adolesc Subst Abuse 2017; 26: 353-66.

6 Gilbody S, Peckham E, Bailey D, et al. Smoking cessation for people with severe mental illness (SCIMITAR+): a pragmatic randomised controlled trial. The lancet Psychiatry 2019; 6: 379-90.

7 Sullivan LE, Fiellin DA, O'Connor PG. The prevalence and impact of alcohol problems in major depression: a systematic review. Am J Med 2005; 118: 330-41.

8 Koskinen J, Löhönen J, Koponen H, Isohanni M, Miettunen J. Prevalence of alcohol use disorders in schizophrenia--a systematic review and meta-analysis. Acta Psychiatr Scand 2009; 120: 85-96.

9 Weinberger AH, Kashan RS, Shpigel DM, et al. Depression and cigarette smoking behavior: A critical review of population-based studies. Am J Drug Alcohol Abuse 2017; 43: 416-31.

10 Thapar A, Collishaw S, Pine DS, Thapar AK. Depression in adolescence. Lancet 2012; 379: $1056-67$. 